\title{
Star formation in a high-pressure environment: An SMA view of the dust ridge
}

\author{
Daniel L. Walker ${ }^{1,2}$ \\ CMZoom Survey Group (PIs: Eric Keto \& Cara Battersby) ${ }^{2}$ \\ ${ }^{1}$ Astrophysics Research Institute, Liverpool John Moores University, \\ IC2, 146 Brownlow Hill, Liverpool, L3 5RF United Kingdom \\ email: D.L.Walker@2009.1jmu.ac.uk \\ ${ }^{2}$ Harvard-Smithsonian Center for Astrophysics, \\ 60 Garden Street, Cambridge, MA 02138, USA \\ email: daniel.walker@cfa.harvard.edu \\ CMZoom: https://www.cfa.harvard.edu/sma/LargeScale/CMZ
}

\begin{abstract}
The star formation rate in the Central Molecular Zone is an order of magnitude lower than in the disk of the Galaxy, given the amount of dense gas there. Understanding why star formation is different in this region is crucial if we are to understand the environmental dependence of star formation. Here, we present the detection of high-mass cores in the CMZ's 'dust ridge' that have been discovered with the Submillimeter Array. These cores range in mass from $\sim 50-1800 \mathrm{M}_{\odot}$ within radii of $0.1-0.25 \mathrm{pc}$. All are young (pre-UCHII), meaning that they are prime candidates for representing the initial conditions of high-mass stars and sub-clusters. We compare these with high-mass cores and clouds in the Galactic disk and find that they are very similar in terms of their masses and sizes, despite being subjected to external pressures that are several magnitudes greater $\left(\sim 10^{8} \mathrm{~K} \mathrm{~cm}^{-3}\right)$. The fact that $>80 \%$ of these cores do not show any signs of star-forming activity in such a high-pressure environment leads us to conclude that this is further evidence of the critical density for star formation being heightened in the CMZ due to turbulence.
\end{abstract}

Keywords. stars: formation, ISM: general, Galaxy: center, submillimeter

\section{Introduction}

Empirical star formation relations have largely been calibrated using detailed studies of star forming regions in the disk of our own and nearby galaxies. Using observations of nearby star-forming regions, Lada et al. (2012) proposed a gas surface density threshold for star formation of $\sim 10^{4} \mathrm{~cm}^{-3}$, above which stars could form efficiently. However, the regions from which relations like this are drawn are typically very similar and do not probe significantly different environmental conditions.

The conditions in the Galactic centre are known to be extreme compared to the Solar neighbourhood, with densities, gas temperatures and pressures being several factors to orders of magnitude greater (Kruijssen et al. 2014). It therefore seems plausible that the process of star formation may proceed differently there. Indeed, a significant fraction of the gas in the Central Molecular Zone (CMZ; inner $\sim 500$ pc of the Galaxy) lies above a volume density of $\sim 10^{4} \mathrm{~cm}^{-3}$, yet the star formation rate (SFR) in the CMZ is $1-2$ orders of magnitude lower than predicted (Longmore et al. 2013a). Current understanding proposes that this is due to the heightened turbulence in the CMZ, which drives the critical density threshold for star formation to higher values (Kruijssen et al. 2014). 


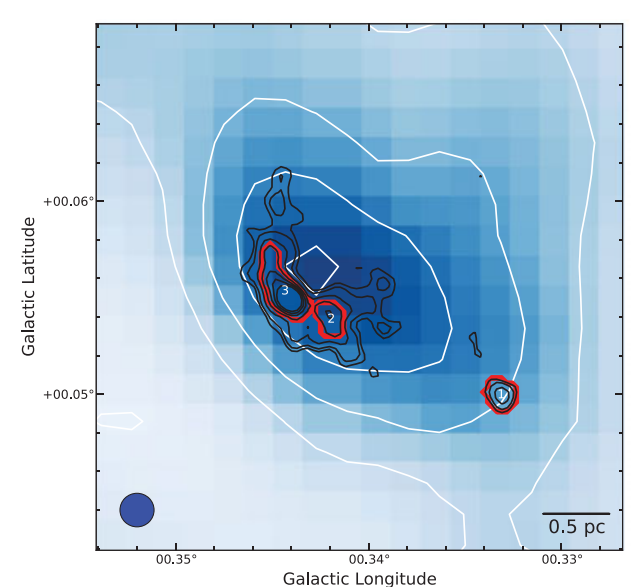

(a)

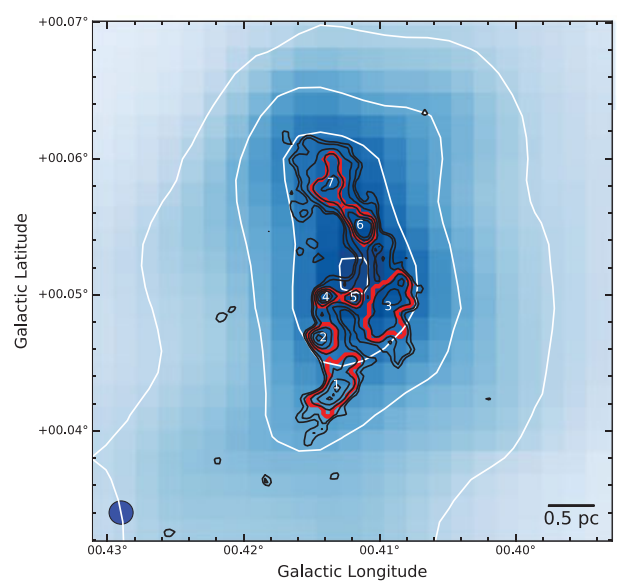

(c)

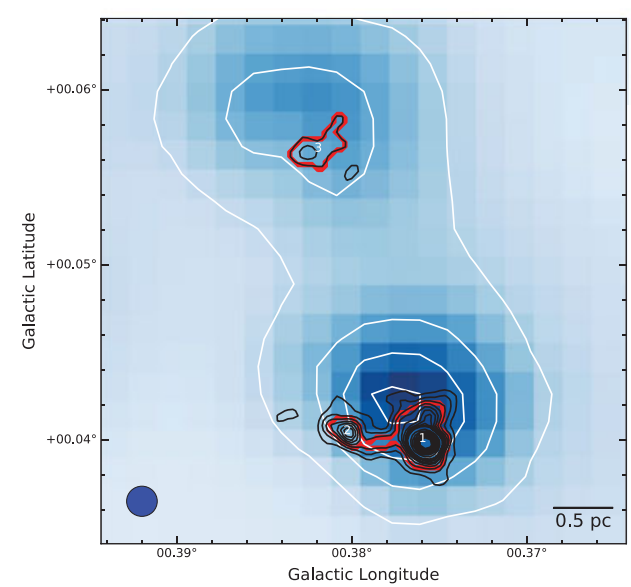

(b)

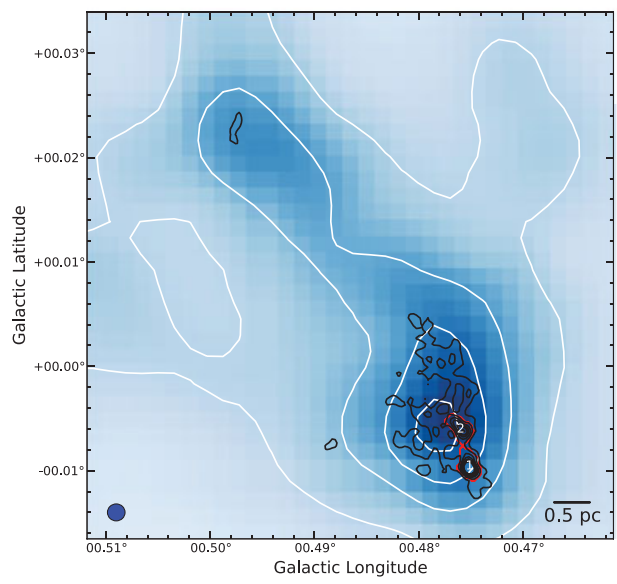

(d)

Figure 1. Top: dust ridge clouds 'b' (left) and 'c' (right). Bottom: dust ridge clouds 'd' (left) and ' $\mathrm{e} / \mathrm{f}$ ' (right). Background image is a Herschel column density map (Battersby et al. in prep.), shown with corresponding white contours. Black contours show the $1.3 \mathrm{~mm}$ continuum emission as seen with the SMA at the 5- $\sigma$ level. Red contours highlight the cores as determined via dendrogram analysis.

\section{The Dust Ridge - quiescent precursors to high-mass clusters}

To understand the process of star formation in the CMZ, it is crucial that we identify and study its unperturbed initial conditions. To achieve this, we target the so-called dust ridge (Lis et al. 1999) - a group of 6 molecular clouds that span $\sim 0.3$ degrees in projection in front of the Galactic Centre, which is at a distance of $8.4 \mathrm{kpc}$ (Reid et al. 2009, 2014). These are G0.253+0.016 (aka 'the Brick'), G0.340+0.055, G0.380+0.050, G0.412+0.052, G0.478-0.005 and G0.496+0.020. For the sake of brevity, we adopt the nomenclature of Lis et al. (1999) and will hereafter refer to these clouds as 'a - f', respectively. They are all $10^{4-5} \mathrm{M}_{\odot}$ with radii $\sim 2-3 \mathrm{pc}$, yet they show no signs of widespread star formation. They are therefore ideal candidates for the initial conditions of star and cluster formation in the CMZ (Longmore et al. 2013b, Longmore et al. 2014, Walker et al. 2015). 


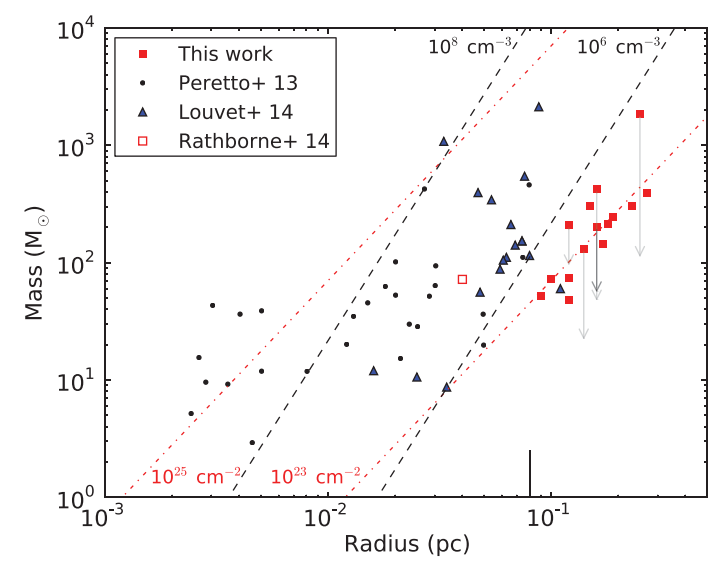

Figure 2. Solid red squares - masses assuming $\mathrm{T}_{d u s t}=20 \mathrm{~K}$ and arrows indicate the range of mass given strong lower-mass limits estimated assuming (where possible) that $\mathrm{T}_{\text {dust }}=\mathrm{T}_{\text {gas }}$. Black points \& blue triangles - high-mass proto-stellar cores in the disk (Peretto et al. 2013, Louvet et al. 2014). Open red square - star-forming core in cloud 'a' (ALMA, Rathborne et al. 2014). Red (dash/dot) - constant surface density, black (dashed) - constant volume density. Solid black vertical line at $0.08 \mathrm{pc}$ - SMA synthesised beam.

To uncover the small-scale, dense structure in these clouds, we observed them as part of our SMA legacy survey of the CMZ - CMZoom † (PIs: E. Keto, C. Battersby). We observed dust ridge clouds 'b-f' in both compact and subcompact configurations at $1.3 \mathrm{~mm}$, achieving $\sim 4^{\prime \prime}$ angular resolution. Figure 1 shows the SMA dust continuum maps. Overall, we detect 15 dense cores in the dust ridge. They have masses ranging from $\sim 50-1800 \mathrm{M}_{\odot}$ with $\mathrm{R} \sim 0.1-0.25$ pc. All are young (pre-UCHis, Immer et al. 2012), meaning that they are prime candidates for representing the initial conditions of high-mass stars and sub-clusters.

\section{Do the properties of high-mass cores vary with environment?}

Having identified this sample of high-mass, compact dust cores in the dust ridge, we compare them to sources in the disk of the Galaxy. To do this we take the samples of highmass cores reported in Peretto et al. (2013) and Louvet et al. (2014), which constitute a large collection of high-mass cores in the Galactic disk from the literature.

In Figure 2 we plot the masses and sizes of our sample of CMZ cores and the cores from disk clouds. Overall we see that the high-mass cores in the CMZ fit within the massradius relation of those in the disk reasonably well - they are not distinctly separated. We note that the cores in our sample are best described by lower volume densities of a few $\times 10^{5}-10^{6} \mathrm{~cm}^{-3}$. Whereas many of the disk sources are at higher volume densities. This may be an effect of the spatial resolution of our SMA data. Higher resolution data are required to confirm this.

We also compare the environmental conditions, namely the gas pressure, that these CMZ sources are subjected to and compare this with pressures in the disk of the Galaxy. To do this, we follow the analysis of Field et al. (2011), in which they take the sample of clouds from the Galactic Ring Survey (GRS, Jackson et al. 2006) and study them in the context of the virial theorem for a self-gravitating isothermal spherical cloud that is subjected to a uniform external pressure. They note that based upon analysis by Heyer et al. (2009), the clouds are not consistent with simple virial equilibrium. They conclude

$\dagger$ CMZoom website: https://www.cfa.harvard.edu/sma/LargeScale/CMZ 


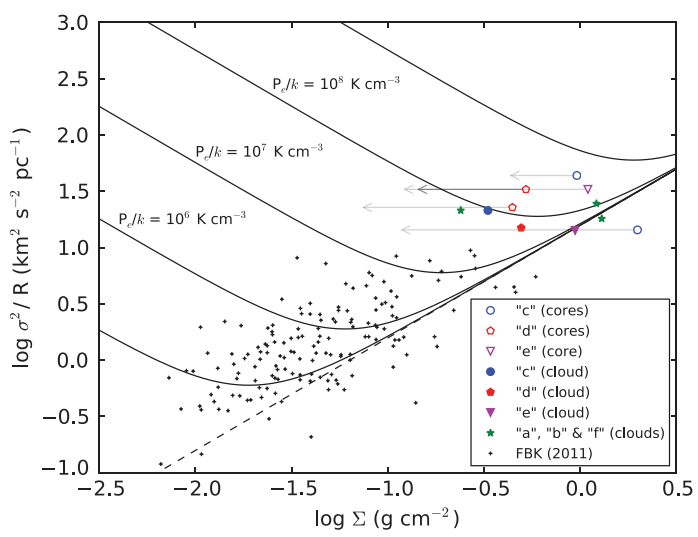

Figure 3. Black crosses - GRS clouds (Heyer et al. 2009). Solid markers - dust ridge clouds, while the open markers represent the core(s) associated with the solid markers. Arrows represent the range in surface densities given our upper and lower mass limits. Curved black lines are those of constant external pressure, while the dashed line is for $\mathrm{P}_{e}=0$.

that this is corrected when accounting for external pressures ranging from $P_{e} / k \sim 10^{4}$ $10^{6} \mathrm{~K} \mathrm{~cm}^{-3}$.

In Figure 3 we replicate the plot given in Figure 3 of Field et al. (2011) and overplot both the dust ridge clouds and their embedded cores that we have detected with the SMA. Note that there are fewer SMA cores displayed in this figure as it requires a measure of the line-width, which was not possible towards all of the dust cores. Figure 3 shows that the external pressures in the CMZ are $\sim 10^{8} \mathrm{~K} \mathrm{~cm}^{-3}-$ up to several orders of magnitude greater than those experienced by the GRS clouds in the Galactic disk.

Comparing Figs. 2 and 3, we see that despite such high external pressures, the embedded cores in the CMZ are comparatively low-density. Out of 15 detected sources, only 2 show tell-tale signs of ongoing star formation, despite being two orders of magnitude more dense than the volume density threshold proposed by Lada et al. (2012) and under pressures that are orders of magnitude greater than in the disk. We conclude that this is further evidence of star formation being inhibited in the CMZ by the heightened turbulent energy, which drives up the critical volume density threshold for star formation in this environment.

\section{References}

Field, G. B., Blackman, E. G., \& Keto, E. R., 2011, MNRAS, 416, 710

Heyer, M., Krawczyk, C., Duval, J. \& Jackson, J. M., 2009, ApJ, 699, 1092

Immer, K., Menten, K. M., Schuller, F. \& Lis, D. C., 2012, A\&̛A, 548, A120

Jackson, J. M., Rathborne, J. M., Shah, R. Y., et al. 2006, ApJS, 163, 145

Kruijssen, J. M. D., Longmore, S. N., Elmegreen, B. G., et al. 2014, MNRAS, 440, 3370

Lada, C. J., Forbrich, J., Lombardi, M. \& Alves, J. F., 2012, ApJ, 745, 190

Lis, D. C., Li, Y., Dowell, C. D. \& Menten, K. M., 1999, ESA Special Publication, Vol. 427, 627

Longmore, S. N., Kruijssen, J. M. D., Bally, J., et al. 2013b, MNRAS, 433, L15

Longmore, S. N., Kruijssen, J. M. D., Bastian, N., et al. 2014, Protostars and Planets VI, 291-314

Louvet, F., Motte, F., Hennebelle, P., et al. 2014, A\&A, 570, A15

Peretto, N., Fuller, G. A., Duarte-Cabral, A., et al. 2013, A\& A, 555, A112

Rathborne, J. M., Longmore, S. N., Jackson, J. M., et al. 2014b, ApJ, 795, L25

Reid, M. J., Menten, K. M., Zheng, X. W., et al. 2009, ApJ, 700, 137

Reid, M. J., Menten, K. M., Brunthaler, A., et al. 2014, ApJ, 783, 130

Walker, D. L., Longmore, S. N., Bastian, N., et al. 2015, MNRAS, 449, 715 